\title{
Classical Intertwiner Space and Quantization
}

\author{
Ping $\mathrm{Xu}^{\star}$ \\ Mathematical Sciences Research Institute, 1000 Centennial Drive, Berkeley, CA 94720, USA, and \\ Department of Mathematics, University of Pennsylvania, Philadelphia, PA 19104, USA. email: \\ ping@math.upenn.edu
}

Received: 15 April 1993/in revised form: 15 November 1993

\begin{abstract}
Given two symplectic realizations, a symplectic manifold called the classical intertwiner space is introduced as a classical analogue of an intertwiner space of representations of an associative algebra. We describe explicitly how a quantum data on realizations induces a quantum data on their classical intertwiner space.
\end{abstract}

\section{Introduction}

Let $G$ be a compact Lie group and $X$ a Hamiltonian $G$-space. $G$ is thus considered as a symmetry group of the classical space $X$. According to the "creed" of geometric quantization, if the classical space $X$ is quantized to a quantum phase space (i.e., a Hilbert space), $G$ becomes a symmetry group of the corresponding quantum space. This idea has in fact inspired many significant results in mathematical physics, among them orbit method of group representations and theory of geometric quantization $[9,19]$.

The intertwiner space between two representations $\rho_{1}: G \rightarrow \operatorname{End}\left(V_{1}\right)$ and $\rho_{2}: G \rightarrow \operatorname{End}\left(V_{2}\right)$ is, by definition, $\operatorname{Hom}_{G}\left(V_{2}, V_{1}\right)$, the space of all $G$-equivariant linear maps from $V_{2}$ to $V_{1}$. When $\rho_{1}$ is irreducible, the dimension of $\operatorname{Hom}_{G}\left(V_{2}, V_{1}\right)$ is usually called the multiplicity of $\rho_{1}$ in $\rho_{2}$, a fundamental concept in representation theory. The classical counterpart of $\operatorname{Hom}_{G}\left(V_{2}, V_{1}\right)$ is closely related to the so-called Marsden-Weinstein reduction [16]. In fact, the classical intertwiner space, for any symplectic homogeneous spaces $X_{1}$ and $X_{2}$, is defined as the symplectic (or Marsden-Weinstein) reduced space $\left(\overline{X_{2}} \times X_{1}\right)_{0}$. A remarkable result of Guillemin-Sternberg [5] asserts that for a Kähler manifold, the geometric quantization of classical intertwiner space is isomorphic to the intertwiner space of the corresponding representations.

\footnotetext{
* Research partially supported by NSF Grant DMS92-03398 and at MSRI supported by NSF Grant DMS90-22140
} 
Recently, more and more interest has been drawn to the so-called hidden symmetry due to the rapid development of quantum group theory. In this case, on the quantum level, the quantum symmetry is represented by a representation of a quantum group, while the classical symmetry is played by a Poisson group homogeneous space. It is therefore desirable to extend Guillemin-Sternberg theory to this more general setting.

To approach this, we shall put this problem into a slightly more general framework. Recall that a Hamiltonian $G$-space $X$ corresponds to an equivariant momentum map $J: X \rightarrow \mathfrak{g}^{*} . J$ is in fact a Poisson map if $\mathrm{g}^{*}$ is equipped with the natural Lie-Poisson structure. Moreover, such a Poisson map uniquely determines the $G$-action on $X$. Such a pair $(X, J)$ is also called a symplectic realization of the Lie-Poisson space $g^{*}$. For a general Poisson manifold $P$, a symplectic realization $(X, J)$ is defined as a symplectic manifold $X$ together with a Poisson map $J: X \rightarrow P$ which is complete in the sense that the pullback to $X$ of every compactly supported function on $P$ has a complete Hamiltonian vector field ${ }^{1}$. As described early in this introduction, a symplectic realization of a Lie-Poisson space $\mathrm{g}^{*}$ is the classical analogue of a representation of the group $G$ or, what is nearly equivalent, of the universal enveloping algebra $U(\mathfrak{g})$. Since $U(\mathfrak{g})$ may be thought of as a quantum deformation of the Poisson algebra of (polynomial) functions on $\mathrm{g}^{*}$, it is a natural generalization of this situation to consider realizations of a general Poisson manifold $P$ as the classical analogues of representations of a quantum deformation of the functions on $P$.

The following table should be useful for understanding the relationship between various notions in this general setting:

\section{CLASSICAL}

symplectic groupoids of Poisson manifolds $\rightarrow$

symplectic realizations of Poisson manifolds $\rightarrow$ representations of associative algebras

\section{QUANTUM}

associative algebras

\section{classical intertwiner space $\quad \rightarrow \quad$ intertwiner of representations}

The horizontal arrows represent processes generally known as "quantization." Our purpose is to extend as much as possible the Guillemin-Sternberg theory to this general non-linear framework. This paper consists of our first attempt toward this direction.

To any two symplectic realizations $J_{1}: X_{1} \rightarrow P$ and $J_{2}: X_{2} \rightarrow P$, there associates in general a symplectic manifold which is a certain quotient space of $\left(\overline{X_{2}} * X_{1}\right)$. This symplectic manifold is called the classical intertwiner space. Here, $\overline{X_{2}} * X_{1}$ denotes the inverse image of the diagonal in $P \times P$ under the map $J_{1} \times J_{2}$, and the bar means reversing the sign of the symplectic structure. This classical intertwiner space is closely related to the symplectic groupoid $\Gamma$ of $P$, and in fact is the quotient space $\Gamma \backslash\left(\overline{X_{2}} * X_{1}\right)$ under the diagonal $\Gamma$-action (see Sect. 4 for the precise definition). When $P$ is the Lie-Poisson $\mathfrak{g}^{*}$ (or even dual of a general Poisson group $G^{*}$ ), this space reduces to the usual intertwiner space [5]. In this paper, we shall describe explicitly how a quantum data on the symplectic groupoid and those on the realizations $X_{1}, X_{2}$ induce a quantum data on the classical intertwiner space

\footnotetext{
${ }^{1}$ Note that in some previous literature $[2,26,28]$, they are called complete symplectic realizations while the terminology of symplectic realizations refers to Poisson maps $J: X \rightarrow P$ without any assumption on completeness.
} 
$\Gamma \backslash\left(\overline{X_{2}} * X_{1}\right)$. Here, as usual, by a quantum data on a symplectic manifold, we mean the following two structures: prequantum circle-bundle and polarization.

The paper is organized as follows.

In Sect. 2, we present some basic facts regarding central extensions of Lie algebroids.

Section 3 concerns central extensions of symplectic groupoids.

Section 4 introduces symplectic realizations and classical intertwiner spaces.

Section 5 is devoted to the discussion on prequantization of symplectic realizations. In particular, we prove that the symplectic groupoid central extension described in Sect. 3 naturally acts on the prequantum bundle of any realization. This result will be needed for the construction in Sect. 6 .

Section 6 contains the main result of this paper, where an explicit construction for the prequantum bundle of a classical intertwiner space is given.

Section 7 is devoted to the discussion on the aspect of polarizations. Finally, some discussions are outlined in Sect. 8.

We would like to thank Jiang-hua Lu and Alan Weinstein for their helpful advice in the course of this work.

\section{Central Extensions of a Lie Algebroid}

The notion of Lie algebroids is a natural generalization of that of Lie algebras. In fact, there are many notions and theorems for Lie algebroids which are parallel to those for Lie algebras. Let us first recall the definition of a Lie algebroid.

Definition 2.1. A Lie algebroid over a manifold $P$ is a vector bundle $\pi: A \rightarrow P$ together with a vector bundle map $\rho: A \rightarrow T P$, called the anchor of $A$, and a Lie algebra structure on $\Gamma(A)$, the space of sections of $A$, satisfying

1. $\rho([X, Y])=[\rho(X), \rho(Y)], X, Y \in \Gamma(A)$;

2. $[X, f Y]=f[X, Y]+\rho(X)(f) Y, X, Y \in \Gamma(A), f \in C^{\infty}(P)$.

Let $A \rightarrow P$ be a Lie algebroid with anchor $\rho$. Suppose that $\xi$ is a Lie algebroid 2-cocycle [14], i.e., a section of the vector bundle $\wedge^{2} A^{*}$ satisfying the condition that

$$
\begin{aligned}
\rho(X) \xi(Y, Z)+\rho(Y) \xi(Z, X) & +\rho(Z) \xi(X, Y)-\xi([X, Y], Z)-\xi([Z, X], Y) \\
& -\xi([Y, Z], X)=0,
\end{aligned}
$$

where $A^{*}$ is the dual bundle of $A$. Let $\tilde{A}=A \oplus(P \times \mathbb{R})$, the vector bundle direct sum of $A$ with the trivial vector bundle $P \times \mathbb{R} \rightarrow P$. It is clear that any section of $\tilde{A}$ can be identified with a pair $(X, f)$, where $X$ is a section of $A$ and $f$ a function on $P$. Define $\tilde{\rho}: \tilde{A} \rightarrow T P$ by $\tilde{\rho}(a, t)=\rho(a)$, for any $a \in A$ and $t \in \mathbb{R}$. Also, we define the bracket of sections of $\tilde{A}$ by

$$
[(X, f),(Y, g)]=([X, Y], \rho(X) g-\rho(Y) f+\xi(X, Y)),
$$

for any $X, Y \in \Gamma(A)$, and $f, g \in C^{\infty}(P)$. It is easy to check that $\tilde{A}$ becomes a Lie algebroid, which we shall call the central extension of the Lie algebroid $A$ by the cocycle $\xi$. In particular, if $\xi=0$, then the bracket of $\tilde{A}$ is defined by

$$
[(X, f),(Y, g)]=([X, Y], \rho(X) g-\rho(Y) f) .
$$


Such an extension is called the trivial extension of $A$ by $\mathbb{R}$. In this case, the natural embedding $i: A \rightarrow \tilde{A}$ given by $i(a)=(a, 0)$ is clearly a Lie algebroid morphism in the sense of [7] (see [15] also).

The proof of the following proposition is straightforward.

Proposition 2.1. If the 2-cocycle $\xi$ is a coboundary $\xi=d \sigma$ for some $\sigma \in \Gamma\left(A^{*}\right)$, i.e.,

$$
\xi(X, Y)=\rho(X) \sigma(Y)-\rho(Y) \sigma(X)-\sigma([X, Y]),
$$

the central extension by $\xi$ is then isomorphic to the trivial central extension under the map:

$$
\phi: \tilde{A} \rightarrow A \oplus(P \times \mathbb{R}), \quad \phi(a, t)=(a, t+\sigma(a)),
$$

where $a \in A, t \in \mathbb{R}$.

Remark. It is well-known that the dual bundle of a Lie algebroid naturally carries a Poisson structure which is called Lie-Poisson structure [2]. The Lie-Poisson structure of a central extension of a Lie algebra is closely related to affine Poisson structures. Therefore it should be quite interesting to investigate in detail what kind of Poisson structures the dual of a central extension of a Lie algebroid corresponds to.

Below is the very example in which we are most interested in this paper.

Let $P$ be a Poisson manifold with Poisson tensor $\pi$. The cotangent bundle $T^{*} P \rightarrow P$ carries a natural Lie algebroid structure [2], which can be described as follows. Given $f \in C \infty(P)$, denote the Hamiltonian vector field corresponding to $f$ by $X_{f}$. Then the anchor $\rho=\pi^{\sharp}: T^{*} P \rightarrow T P$ is determined by $\rho(f d g)=f X_{g}$. Given $\omega, \theta \in \Omega^{1}(P)$, and writing $X_{\omega}=\rho \omega$ and $X_{\theta}=\rho \theta$, the Lie algebroid bracket is

$$
\{\omega, \theta\}=L_{X_{\omega}} \theta-L_{X_{\theta}} \omega-d[\pi(\omega, \theta)] .
$$

The Poisson tensor $\pi$ can be considered as a Lie algebroid 2-cocycle in a natural way $[8,24]$. Therefore, there associates a canonical central extension $\tilde{A}=$ $T^{*} P \oplus(P \times \mathbb{R})$. The global object corresponding to $\tilde{A}$ is closely related to the prequantization of the symplectic groupoid, which is to be described in the following section.

Remark. Lu has observed that this 2-cocycle is a coboundary iff there is a vector field $V$ on $P$ such that $\pi=[V, \pi]=L_{V} \pi$. In other words, $V$ is a Liouville vector field of the Poisson manifold $P$. Thus according to Proposition 2.1, the existence of a Liouville vector field is equivalent to saying that $\tilde{A}$ is isomorphic to the trivial central extension. It is easily seen that any Lie-Poisson space $g^{*}$ possesses such a property.

\section{Symplectic Groupoids and Prequantization}

The global objects corresponding to Lie algebroids are the so-called Lie groupoids. Briefly, a groupoid is a small category whose morphisms are all invertible. Below is the precise definition. We refer the reader to [14] for more details on Lie groupoid theory.

Definition 3.1. A Lie groupoid $\Gamma$ over a manifold $P$ is a pair $(\Gamma, P)$ equipped with surjective submersions $\alpha, \beta: \Gamma \rightarrow P$ (called the source and target maps respectively); a multiplication map $m$ from $\Gamma_{2}=\{(g, h) \in \Gamma \times \Gamma \mid \beta(g)=\alpha(h)\}$ to $\Gamma$, an injection $\varepsilon: P \rightarrow \Gamma$ (called the unit map); and an inverse mapping $i: \Gamma \rightarrow \Gamma$ satisfying (where $m(g, h)$ is denoted by $g h$, and $i(g)$ by $\left.g^{-1}\right)$ : 
1. (associativity) $g(h k)=(g h) k$, which means that if one side is defined, so is the other and they are equal;

2. (identities) $\varepsilon(\alpha(g)) g=g \varepsilon(\beta(g))$;

3. (inverses) $g g^{-1}=\varepsilon(\alpha(g))$ and $g^{-1} g=\varepsilon(\beta(g))$.

We usually use the notation $(\Gamma \rightrightarrows P, \alpha, \beta)$ to denote such a Lie groupoid.

Associated to any Lie groupoid, there is a Lie algebroid as an infinitesimal invariant. However, conversely, a Lie algebroid may not always be the Lie algebroid of a Lie groupoid. In the case that it does arise from a Lie groupoid, one says that the Lie algebroid is integrable [14].

In particular, a Lie groupoid corresponding to the Lie algebroid arising from a Poisson manifold as described by Eq. (1) usually carries a symplectic structure, which is compatible with the groupoid structure in a certain sense. Such a groupoid is called a symplectic groupoid. More precisely, a symplectic groupoid is a Lie groupoid $(\Gamma \rightrightarrows P, \alpha, \beta)$ with a symplectic structure on $\Gamma$ such that the graph of multiplication $\Lambda=\{(g, h, g h) \mid \beta(g)=\alpha(h)\}$ is a lagrangian submanifold of $\Gamma \times \Gamma \times \bar{\Gamma}$ (the bar denotes reversing the sign of the symplectic structure). For more details on symplectic groupoids, see [2,21].

A main result of [24] asserts that the canonical central extension $\tilde{A}=$ $T^{*} P \oplus(P \times \mathbb{R})$ as described at the end of Sect. 2 is integrable (i.e. admits a global groupoid) provided that the Poisson manifold $P$ admits a symplectic groupoid.

\section{Theorem 3.1 [24].}

1. If the symplectic groupoid $\left(\Gamma \rightrightarrows P, \alpha_{0}, \beta_{0}\right)$ is $\alpha$-simply connected, and prequantizable, then there exists a unique prequantization $E$ of $\Gamma$ such that the identity space $P$ has no holonomy.

2. Let $E \stackrel{\pi}{\rightarrow} \Gamma$ be such a prequantization with connection $\theta$ so that $E$ has no holonomy over $\varepsilon_{0}(P)$, where $\varepsilon_{0}: P \rightarrow \Gamma$ is the unit map. Let $\varepsilon: \varepsilon_{0}(P) \rightarrow E$ be any horizontal section over $\varepsilon_{0}(P)$; then there exists a canonical groupoid structure on $E$ over $P$, with unit map $\varepsilon \circ \varepsilon_{0}$, source $\alpha=\alpha_{0} \circ \pi$, and target $\beta=\beta_{0} \circ \pi$, such that $\pi: E \rightarrow \Gamma$ is a groupoid homomorphism.

3. The Lie algebroid of $(E \rightrightarrows P, \alpha, \beta)$ is isomorphic to the canonical central extension $\widetilde{A}=T^{*} P \oplus(P \times \mathbb{R})$ introduced in Sect. 2 .

$E$ is called the canonical central extension of the symplectic groupoid $\Gamma$.

\section{Symplectic Realizations and Classical Intertwiner Spaces}

A symplectic realization $(X, J)$ of a Poisson manifold $P$ is defined as a symplectic manifold $X$ together with a Poisson map $J: X \rightarrow P$ which is complete in the sense that the pullback to $X$ of every compactly supported function on $P$ has a complete Hamiltonian vector field. Symplectic realizations are generally considered as classical analogue of representations of an associative algebra. Morphisms between realizations are, by definition, lagrangian submanifolds of $\overline{X_{2}} \times X_{1}$ (the bar denotes reversing the sign of the symplectic structure) contained in the coisotropic submanifold $\overline{X_{2}} * X_{1}=\left(J_{2} \times J_{1}\right)^{-1}$ (diagonal in $\left.\bar{P} \times P\right)$. We however note that these do not form a category because of the usual clean intersection requirements for good compositions $[27,26]$. 
Now suppose that $P$ is integrable as a Poisson manifold in the sense that it admits an $\alpha$-simply connected symplectic groupoid $\Gamma$. Given such a symplectic groupoid $(\Gamma \rightrightarrows P, \alpha, \beta)$, by a symplectic left $\Gamma$-module, we mean a symplectic manifold $X$ together with a smooth map $J: X \rightarrow P$ such that $X \stackrel{J}{\rightarrow} P$ admits a symplectic left $\Gamma$-action (see $[17,27]$ for the definition of symplectic groupoid action). $J$ is usually called the momentum map of the module $X$. The symplectic left modules of a given symplectic groupoid $\Gamma$ become a "category" $\zeta(\Gamma)$ in the following sense: the objects of $\zeta(\Gamma)$ are symplectic left $\Gamma$-modules, the morphisms are canonical relations satisfying certain conditions compatible with the groupoid actions, and the composition of morphisms is the set-theoretic composition of relations. More precisely, a morphism from a symplectic left $\Gamma$-module $X_{2}$ to a symplectic left $\Gamma$-module $X_{1}$ is a lagrangian submanifold $\mathscr{L} \subset \overline{X_{2}} * X_{1}$ that is invariant under the diagonal action of $\Gamma$. As usual, $\zeta(\Gamma)$ is not a true category and it requires the usual clean intersection assumption for the composition of morphisms being a morphism.

It is shown [26] that symplectic realizations of a Poisson manifold are in fact in one-one correspondence with symplectic left-modules of its $\alpha$-simply connected symplectic groupoid. For this reason, we will not distinguish these two concepts in the sequel.

Theorem 4.1 [26]. Let $\Gamma$ be an $\alpha$-simply connected symplectic groupoid over $P$. If $X$ is a symplectic left $\Gamma$-module, then its momentum map $\rho: X \rightarrow P$ is a symplectic realization. Conversely, any symplectic realization $\rho: X \rightarrow P$ naturally admits a symplectic $\Gamma$-action so that it becomes a symplectic left $\Gamma$-module.

Let $J_{1}: X_{1} \rightarrow P$ and $J_{2}: X_{2} \rightarrow P$ be any two symplectic realizations. For technical reasons, we always assume that, as symplectic left $\Gamma$-modules, $\Gamma$ acts on $X_{1}$ and $X_{2}$ freely and properly, and that the map $J_{2} \times J_{1}: X_{2} \times X_{1} \rightarrow P \times P$ is transversal to the diagonal $\Delta_{P} \subset P \times P$ so that $\Gamma \backslash\left(X_{2} * X_{1}\right)$ is a smooth manifold, where $X_{2} * X_{1}$ denotes the irverse image of the diagonal $\Delta_{P}$ under the map $J_{2} \times J_{1}$. It can be proved that $\Gamma \backslash\left(\overline{X_{2}} * X_{1}\right)$ is a symplectic manifold with the symplectic structure being naturally induced from that on $\overline{X_{1}} \times X_{2}$ (see Prop. 2.1 [27] or Theorem $1[10])$. We shall call this space the classical intertwiner space between the realizations $J_{1}: X_{1} \rightarrow P$ and $J_{2}: X_{2} \rightarrow P$.

Heuristically, as the Poisson manifold $P$ is quantized to an associative algebra $\mathscr{A}$, the realizations $J_{1}: X_{1} \rightarrow P$ and $J_{2}: X_{2} \rightarrow P$ are quantized to representations $\pi_{1}$ and $\pi_{2}$ of the algebra $\mathscr{A}$. Then, the symplectic space $\Gamma \backslash\left(\overline{X_{2}} * X_{1}\right)$ expects to become the intertwiner space of the representations $\pi_{1}$ and $\pi_{2}$. According to the "symplectic creed," a classical "state" is a lagrangian submanifold of the symplectic manifold. The following proposition shows that classical "states" of the symplectic manifold $\Gamma \backslash\left(\overline{X_{2}} * X_{1}\right)$ are indeed the same as morphisms of the realizations. Thus, it gives another rationale for our terminology.

Proposition 4.1. There is a one-one correspondence between morphisms of realizations $J_{1}: X_{1} \rightarrow P, J_{2}: X_{2} \rightarrow P$ and lagrangian submanifolds of the classical intertwiner space $\Gamma \backslash\left(\overline{X_{2}} * X_{1}\right)$.

Proof. Let $\mathscr{L}$ be any morphism of the realizations $J_{1}$ and $J_{2}$, i.e., a lagrangian submanifold of $\overline{X_{2}} * X_{1}$. By Proposition 3.1 in [26], $\mathscr{L}$ is invariant under the diagonal action of $\Gamma$. It is thus simple to see by dimension counting that $\Gamma \backslash \mathscr{L}$ is a lagrangian submanifold of $\Gamma \backslash\left(\overline{X_{2}} * X_{1}\right)$. Conversely, the preimage of any 
lagrangian submanifold of the quotient space $\Gamma \backslash\left(\overline{X_{2}} * X_{1}\right)$ is easily seen to be a lagrangian submanifold sitting inside $\overline{X_{2}} * X_{1}$. term.

For Poisson groups, classical intertwiner spaces can be expressed in a simpler

Suppose that $G$ is a complete simply-connected Poisson group with simply-connected dual $G^{*}[3,13]$. Let $m: G^{*} \times G^{*} \rightarrow G^{*}$ denote the group multiplication. Analogous to the tensor product of representations of a Hopf algebra, there is an operation among symplectic realizations of $G^{*}$, called the "tensor product" [25], which is described as follows. Let $J_{1}: X_{1} \rightarrow G^{*}$ and $J_{2}: X_{2} \rightarrow G^{*}$ be symplectic realizations of $G^{*}$. Then $J_{1} \times J_{2}: X_{1} \times X_{2} \rightarrow G^{*} \times G^{*}$ is a symplectic realization of $G^{*} \times G^{*}$. Since the group multiplication $m: G^{*} \times G^{*} \rightarrow G^{*}$ is a Poisson map, $X_{1} \times X_{2} \stackrel{\stackrel{\circ}{\circ}\left(J_{1} \times J_{2}\right)}{\longrightarrow} G^{*}$ is thus a symplectic realization of $G^{*}$. We write this realization as $X_{1} \otimes X_{2}$, and the realization map $m \circ\left(J_{1} \times J_{2}\right)$ as $J_{1} \otimes J_{2}$. In this way, we obtain an associative product on the set of all realizations of $G^{*}$, which is called the "tensor product."

The $\alpha$-simply connected symplectic groupoid $D$ of the Poisson group $G^{*}$ is diffeomorphic, as a differential manifold, to $G \times G^{*}$, and the groupoid structure is defined in terms of dressing actions [12]. Given a symplectic realization $J: X \rightarrow G^{*}$, there is a symplectic groupoid $D$-action on $X$ as discussed above, as well as a Poisson group $\mathrm{G}$-action as introduced by $\mathrm{Lu}$ [11] by considering $J$ as the momentum mapping. It was shown [25] that these two actions are in fact equivalent in the sense that one action can be expressed naturally in terms of the other. In particular, the Poisson manifold resulting from the groupoid reduction $D \backslash X$ $[27,29,25]$ is Poisson diffeomorphic to the Poisson group reduction $X / G$ as defined by Semenov-Tian-Shansky [18]. The following proposition can also be easily verified.

Proposition 4.2. Let $J_{1}: X_{1} \rightarrow G^{*}$ and $J_{2}: X_{2} \rightarrow G^{*}$ be symplectic realizations of $G^{*}$. Then, the classical intertwiner space $D \backslash\left(\overline{X_{2}} * X_{1}\right)$ is symplectic diffeomorphic to the symplectic reduction $\left(\overline{J_{2}} \otimes J_{1}\right)^{-1}\left(1_{G^{*}}\right) / G($ see $[11]$ for symplectic reduction in the context of Poisson group actions), where $\overline{J_{2}}: \overline{X_{2}} \rightarrow G^{*}$ denotes the inverse realization defined by $\overline{J_{2}}(x)=\left(J_{2}(x)\right)^{-1}$, and $1_{G^{*}}$ is the unit of $G^{*}$.

As a consequence, we conclude that to study classical intertwiner spaces for Poisson groups, it suffices to confine one's attention to the symplectic reduction $J^{-1}\left(1_{G^{*}}\right) / G$ for a certain symplectic realization $J: X \rightarrow G^{*}$.

As an immediate consequence of Proposition 4.1, we have the following result generalizing Theorem 2.6 in [5].

Corollary 4.1. Let $G$ be a simply connected Poisson Lie group with $G^{*}$ being its simply connected dual group. Let $X$ be a symplectic manifold with a Poisson group $G$-action and $J: X \rightarrow G^{*}$ its corresponding momentum mapping [11]. There is a oneone correspondence between $G$-invariant lagrangian submanifolds of $X$ and lagrangian submanifolds of the reduced space $J^{-1}\left(1_{G^{*}}\right) / G$, where $1_{G^{*}}$ is the unit of $G^{*}$.

\section{Prequantization of Symplectic Realizations}

This section can be considered as a continuation of Sect. 2, aimed at the global feature. Since we are mostly interested in the case of symplectic groupoids in the 
present paper, we shall concentrate on the particular example of the central extension arising from Poisson manifolds as described at the end of Sect. 2.

Let $P$ be a Poisson manifold with Poisson tensor $\pi$, and $\left(\Gamma \rightrightarrows P, \alpha_{0}, \beta_{0}\right)$ an $\alpha$-simply connected symplectic groupoid. Let $J: X \rightarrow P$ be a symplectic realization. Suppose that $X$ is prequantizable and $\phi: L \rightarrow X$ is a prequantum circle-bundle of $X$ with connection $\theta_{L}$.

To describe our construction, we first proceed at the level of Lie algebroids. As in Sect. 2, let $\tilde{A}=A \oplus(P \times \mathbb{R})$ be the canonical central extension of $T^{*} P$ corresponding to the Poisson manifold $P$. The connection $\theta_{L}$ thus induces a Lie algebroid action [7] of $\tilde{A}$ on the prequantum circle-bundle $L$, as described by the following:

Proposition 5.1. Let $\tilde{J}=J \circ \phi: L \rightarrow P$ be the composition of $\phi: L \rightarrow X$ with $J: X \rightarrow P$. Then,

$$
(\omega, f) \in \Gamma(\tilde{A}) \rightarrow \hat{X}_{J^{*} \omega}-\left(\tilde{J}^{*} f\right) \zeta \in \mathscr{X}(L)
$$

defines a Lie algebroid action, where $\omega \in \Omega^{1}(P), f \in C^{\infty}(P), \hat{X}_{J^{*} \omega}$ is the horizontal lift of the vector field $X_{J^{*} \omega}$, and $\zeta$ is the Euler vector field on $L$ generating the circle action.

The proof is straightforward and is left to the reader.

As in the case of Lie algebras, one expects to integrate a Lie algebroid action to a Lie groupoid action. This can be done under certain mild conditions. Below, we shall outline this result briefly.

There are in fact two obstructions to such an integration: a dynamical one and a topological one. The dynamical obstruction is, roughly speaking, that certain vector fields involved need to be complete. More precisely, we say that a Lie algebroid action on a manifold $M: \Gamma(A) \rightarrow \mathscr{X}(M), \eta \rightarrow X_{\eta} \in \mathscr{X}(M), \forall \eta \in \Gamma(A)$, is complete if $X_{\eta}$ is complete whenever $\eta$, considered as a left invariant vector field on $\Gamma$, is complete. The topological condition requires a certain assumption on simply-connectivity, which in our case, means that $\Gamma$ is $\alpha$-simply connected.

Proposition 5.2. Let $(\Gamma \rightarrow P, \alpha, \beta)$ be an $\alpha$-connected and simply connected Lie groupoid with Lie algebroid $A \rightarrow P$. Then a Lie groupoid $\Gamma$-action on $J: S \rightarrow P$ corresponds to a complete Lie algebroid action. Conversely, any complete Lie algebroid action on $J: S \rightarrow P$ can be integrated to a Lie groupoid $\Gamma$-action.

This proposition can be proved by imitating the proof of Theorem 3.1 in [26], which is left to the reader.

We now turn to the particular case as in Proposition 5.1. The groupoid corresponding to $\tilde{A}$ is the central extension groupoid $(E \rightrightarrows P, \alpha, \beta)$ as described in Theorem 3.1. Certainly, we expect that this Lie algebroid action extends to a groupoid $E$-action. However, one cannot simply apply Proposition 5.2, since the groupoid $(E \rightrightarrows P, \alpha, \beta)$ is generally not $\alpha$-simply connected. In fact, we have the following

Proposition 5.3. The central extension groupoid $(E \rightrightarrows P, \alpha, \beta)$ is $\alpha$-simply connected iff the cohomology class of $\left.\Omega\right|_{\alpha_{0}^{-1}(u)}$ does not vanish in $H^{2}\left(\alpha_{0}^{-1}(u), \mathbb{R}\right)$ for any $u \in P$, where $\left.\Omega\right|_{\alpha_{0}^{-1}(u)}$ denotes the restriction of the symplectic form $\Omega$ of $\Gamma$ on the fiber $\alpha_{0}^{-1}(u)$.

Proof. For any $u \in P, \alpha^{-1}(u)$ is a principal $T^{1}$-bundle over $\alpha_{0}^{-1}(u)$, with $\left.\Omega\right|_{\alpha_{0}^{-1}(u)}$ being the curvature. The conclusion thus follows immediately by applying Gysin sequence [1]. 
Below, we shall get around this difficulty using the method adopted from [24]. Our main result is the following:

Theorem 5.1. The central extension groupoid $(E \rightrightarrows P, \alpha, \beta)$, naturally acts on $\widetilde{J}=$ $J \circ \phi: L \rightarrow P$, lifting the Lie algebroid action of $\widetilde{A}$ on $L$ as defined in Proposition 5.1.

Let $\mathscr{D}$ be the distribution on $E \times L \times L$ defined by $\mathscr{D}=\left\{\left(\hat{X}_{\alpha_{0}^{*} \omega}-\left(\alpha^{*} f\right) \xi, 0\right.\right.$, $\left.\left.\hat{X}_{J^{*} \omega}-\left(\widetilde{J}^{*} f\right) \zeta\right) \mid \omega \in \Omega^{1}(P), f \in C^{\infty}(P)\right\}$. Here $\xi$ and $\zeta$, respectively, denote the vector fields on $E$ and $L$ generating their circle actions. Let $I$ be the submanifold of $E \times L \times L$ consisting of all elements of the form $(\widetilde{J}(l), l, l)$ for any $l \in L$, where $P$ is considered as a submanifold of $E$ via the embedding $\varepsilon \circ \varepsilon_{0}$. I is clearly transversal to the distribution $\mathscr{D}$. Let $\Xi$ be the minimal $\mathscr{D}$-invariant submanifold containing $I$ and immersed in $E \times L \times L$ obtained by the method of characteristics.

It suffices to show that $\Xi$ is a graph over the submanifold $E_{2}=\{(\kappa, l) \in E \times$ $L \mid \beta(\kappa)=\widetilde{J}(l)\}$. Obviously, the image of $\Xi$ under the natural projection $\rho: E \times L \times$ $L \rightarrow E \times L$ given by $\rho(\kappa, l, m)=(\kappa, l)$, is $E_{2}$. To prove the other half, we note that the two-torus $T^{2}$ naturally acts on $E \times L \times L$ by

$$
(s, t) \cdot(\kappa, l, m)=(s \cdot \kappa, t \cdot l,(s+t) \cdot m), \quad \text { for any }(\kappa, l, m) \in E \times L \times L \text { and } s, t \in T^{1} .
$$

Thus, $(E \times L \times L) / T^{2} \stackrel{p}{\rightarrow} \Gamma \times X \times X$ with $p([\kappa, l, m])=(\pi(\kappa), \phi(l), \phi(m))$ is a circle bundle, which is in fact a prequantization of $\Gamma \times X \times \bar{X}$, with connection $\bar{\Theta}$. The latter is the one-form on $(E \times L \times L) / T^{2}$ naturally induced from $\Theta=\left(\theta, \theta_{L},-\theta_{L}\right) \in$ $\Omega^{1}(E \times L \times L)$.

Below, we shall characterize some basic properties of the manifold $\Xi$ in the following series of lemmas.

Lemma 5.1. The $T^{2}$-action leaves $\Xi$ invariant.

Proof. Since $(\zeta, 0, \zeta) \in \mathscr{D}$, then $(\kappa, l, m) \in \Xi$ iff $(t \cdot \kappa, l, t \cdot m) \in \Xi$ for any $t \in T^{1}$. For the action of the other generator of $T^{2}$, we note that, by definition, $(\kappa, l, m) \in \Xi$ iff $\kappa=\Phi^{\alpha}(u)$, and $m=\Phi^{\tilde{J}}(l)$, where $u=\beta(\kappa)=\widetilde{J}(l) \in P, \Phi^{\alpha}$ is a product of flows on $E$ generated by vector fields of the form $\hat{X}_{\alpha_{0}^{*} \omega}-\left(\alpha^{*} f\right) \xi$ for $\omega \in \Omega^{1}(P)$ and $f \in C^{\infty}(P)$, and $\Phi^{\tilde{J}}$ is the corresponding product of flows on $L$, in the same order, generated by $\hat{X}_{J^{*} \omega}-\left(\widetilde{J}^{*} f\right) \zeta$. Since $\hat{X}_{J^{*} \omega}-(\widetilde{J} * f) \zeta$ commutes with the Euler vector field $\zeta$ for any $(\omega, f) \in \Gamma(\tilde{A})$, it follows that $t \cdot m=t \cdot \Phi^{\tilde{J}}(l)=\Phi^{\tilde{J}}(t \cdot l)$ for any $t \in T^{1}$. Therefore, $(\kappa, t \cdot l, t \cdot m) \in \Xi$ iff $(\kappa, l, m) \in \Xi$.

Lemma 5.2. The pull back of $\Theta$ on $\Xi$ is zero.

Proof. We denote by $\lambda$ the natural projection $E \times L \times L \rightarrow \Gamma \times X \times X$, and write $\Delta=\lambda^{-1}(\Lambda)$, where $\Lambda \subset \Gamma \times X \times X$ is the graph of the groupoid action. Let $i$ be the natural inclusion from $\Delta$ to $E \times L \times L$. It is easy to check that $d\left(i^{*} \Theta\right)=0$ and $i^{*} \Theta$ is nowhere zero in $\Delta$. Hence, the equation $i^{*} \Theta=0$ defines a codimension one foliation $\mathscr{F}$ on $\Delta$.

Any tangent vector to $I$ has the form $((T \tilde{J}) v, v, v)$, where $v$ is a tangent vector of $L$. Now,

$$
\begin{aligned}
((T \tilde{J}) v, v, v)\lrcorner i^{*} \Theta & \left.=(T \tilde{J}) v\lrcorner \theta+v\lrcorner \theta_{L}-v\right\lrcorner \theta_{L} \\
& =(T \tilde{J}) v\lrcorner \theta \\
& =0,
\end{aligned}
$$

where the last step follows from the fact that the image of the unit map $\varepsilon^{\circ} \varepsilon_{0}: P \rightarrow E$ is a horizontal section. Therefore,

$$
\left.i^{*} \Theta\right|_{I}=0
$$


Obviously, $I$ is connected if $P$ is (otherwise, we simply consider $P$ component by component). Let $\mathscr{L}$ be the maximal integral submanifold of $\mathscr{F}$ containing $I$. In order to prove the lemma, it suffices to show that

$$
\Xi \subseteq \mathscr{L}
$$

For this, we only need to show that $\mathscr{D} \subseteq \mathscr{F}$ as a distribution. This, however, easily follows from the following computation:

$$
\begin{aligned}
& \left.\left(\hat{X}_{\alpha_{0}^{*} \omega}-\left(\alpha^{*} f\right) \xi, 0, \hat{X}_{J^{*} \omega}-\left(\tilde{J}^{*} f\right) \zeta\right)\right\lrcorner\left(\theta, \theta_{L},-\theta_{L}\right) \\
= & -\alpha^{*} f+\tilde{J}^{*} f \\
= & 0,
\end{aligned}
$$

where the last step uses the fact that $\widetilde{J}(m)=\alpha(\kappa)$ for any $(\kappa, l, m) \in \Xi$.

We can now turn to the proof of Theorem 5.1.

Proof of Theorem 5.1. It suffices to show that $\Xi$ is a graph over $E_{2}=\{(\kappa, l) \in E \times$ $L \mid \beta(\kappa)=\widetilde{J}(l)\}$. Obviously, the image of $\Xi$ under the projection $\rho: E \times L \times L \rightarrow$ $E \times L, \rho(\kappa, l, m)=(\kappa, l)$, is $E_{2}$. To prove the other half, for any $x \in X$, we let $\Lambda_{x}=\left\{(r, x, r \cdot x) \mid \forall r \in \Gamma\right.$ such that $\left.\beta_{0}(r)=J(x)\right\} \subset \Lambda$. Then, $\Lambda_{x}$ is diffeomorphic to $\beta_{0}^{-1}(J(x))$, hence is simply connected. Therefore, it is clear that $p^{-1}\left(\Lambda_{x}\right) \rightarrow \Lambda_{x}$ is a flat bundle without holonomy. On the other hand, it follows from Lemma 5.1 and 5.2 that $p^{-1}\left(\Lambda_{x}\right) \cap\left(\Xi / T^{2}\right)$ is a connected submanifold on which the restriction of $\bar{\Theta}$ vanishes. Hence, $p^{-1}\left(\Lambda_{x}\right) \cap\left(\Xi / T^{2}\right)$ is a horizontal section over $\Lambda_{x}$, and therefore is diffeomorphic to $\Lambda_{x}$. Suppose that $\left(\kappa, l, m_{1}\right)$ and $\left(\kappa, l, m_{2}\right) \in \Xi$. Since $\left[\kappa, l, m_{1}\right]$ and $\left[\kappa, l, m_{2}\right] \in p^{-1}\left(\Lambda_{\phi(l)}\right) \cap\left(\Xi / T^{2}\right)$ and have the same image under the map $p$, then $\left[\kappa, l, m_{1}\right]=\left[\kappa, l, m_{2}\right]$. It thus follows that $\left(\kappa, l, m_{1}\right)=(s, t) \cdot\left(\kappa, l, m_{2}\right)$ for some $(s, t) \in$ $T^{2}$, which immediately implies that $m_{1}=m_{2}$. This completes the proof.

Remark. (1) It is easy to see that $\Xi / T^{2}$ is in fact a horizontal section over the graph of groupoid action $\Lambda$. In other words, $\Lambda$ has no holonomy under the circle bundle $p:(E \times L \times L) / T^{2} \rightarrow \Gamma \times X \times X$.

(2) It is worth noting that the symplectic groupoid $\Gamma$ itself does not act on the circle-bundle $L$ in general. In fact, on the Lie algebroid level, whether an action exists depends on whether the Lie algebroid $A=T^{*} P$ can be embedded as a subalgebroid of the canonical central extension $\tilde{A}=T^{*} P \oplus(P \times \mathbb{R})$. The latter is, however, related to the existence of a Liouville vector field on the Poisson manifold $P$, as pointed out by $\mathrm{Lu}$ (see the remark at the end of Sect. 2).

\section{Prequantization of the Classical Intertwiner Space}

The purpose of this section is to give an explicit construction of the prequantum bundle for the classical intertwiner space, using the prequantum bundles of the realizations and the symplectic groupoid.

First, we need a couple of lemmas. The first one is a direct consequence of Theorem 5.1.

Lemma 6.1. Under the same assumption as in Theorem 5.1, then

(1) the projection $\lambda=(\pi, \phi, \phi): E \times L \times L \rightarrow \Gamma \times X \times X$ maps the graph of groupoid action $\Xi \subset E \times L \times L$ to the graph of groupoid action $\Lambda \subset \Lambda \times X \times X$. I.e., for any $\tau \in E$ 
and $w \in L$ such that $\beta(\tau)=\widetilde{J}(w)$, we have

$$
\phi(\tau \cdot w)=\pi(\tau) \cdot \phi(w) ;
$$

(2) on $L$, the groupoid E-action commutes with the circle-action.

Proof. To prove (1), let $\mathscr{D}$ be the distribution in $E \times L \times L$ as defined in the previous section. It is simple to note that $\lambda$ pushes down any vector in $\mathscr{D}$ to a vector tangent to the graph $\Lambda$. Also, it is trivial to see that $\lambda$ sends $I$ to a submanifold of $\Lambda$. The conclusion thus follows immediately from the construction of $\Xi$.

For (2), we note that infinitesimally the groupoid $E$-action on $L$ is generated by vector fields of the form $\hat{X}_{J^{*} \omega}-\left(\tilde{J}^{*} f\right) \zeta$ for $\omega \in \Omega^{1}(P)$ and $f \in C^{\infty}(P)$ (cf. Proposition 5.1). It is easy to check that any vector field of this form commutes with $\zeta$. This concludes the proof of the lemma.

Lemma 6.2. Under the same assumption as in Theorem 5.1, $\theta_{L}$ is invariant under the groupoid E-action. I.e., for any $\tau \in E$ such that $\beta(\tau)=u$ and $\alpha(\tau)=v$,

$$
\Phi_{\tau}^{*}\left(\left.\theta_{L}\right|_{u}\right)=\left.\theta_{L}\right|_{v},
$$

where $\left.\theta_{L}\right|_{u}$ and $\left.\theta_{L}\right|_{v}$ are the restrictions of $\theta_{L}$ on the fibers $\phi^{-1}(u)$ and $\phi^{-1}(v)$ respectively, and $\Phi_{\tau}: \phi^{-1}(u) \rightarrow \phi^{-1}(v)$ denotes the diffeomorphism of $\phi$-fibers multiplying by the groupoid element $\tau$.

Proof. For any $(\omega, f) \in \Gamma(\tilde{A})$, we have

$$
\begin{aligned}
L_{\hat{X}_{J^{*} \omega}-\left(\tilde{J}^{*} f\right) \zeta} \theta_{L} & \left.\left.=\left(\hat{X}_{J^{*} \omega}-\left(\tilde{J}^{*} f\right) \zeta\right)\right\lrcorner d \theta_{L}+d\left[\left(\hat{X}_{J^{*} \omega}-\left(\tilde{J}^{*} f\right) \zeta\right)\right\lrcorner \theta_{L}\right] \\
& \left.=\left(\hat{X}_{J^{*} \omega}-\left(\tilde{J}^{*} f\right) \zeta\right)\right\lrcorner \phi^{*} \Omega_{X}+d\left(-\tilde{J}^{*} f\right) \\
& \left.=\hat{X}_{J^{*} \omega}\right\lrcorner \phi^{*} \Omega_{X}-d\left(\tilde{J}^{*} f\right) \\
& =\tilde{J}^{*}(\omega-d f),
\end{aligned}
$$

where $\Omega_{X}$ is the symplectic form on $X$.

It thus follows that $L_{\hat{X}_{J^{*} \omega}-\left(\tilde{J}^{*} f\right) \zeta} \theta_{L}=0$, iff $\omega=d f$. Therefore, the flow of the vector field $\hat{X}_{J^{*} d f}-\left(\tilde{J}^{*} f\right) \zeta$ preserves the connection form $\theta_{L}$. Since any point $\tau \in E$ can be reached by a product of flows generated by such vector fields, it thus follows that $\Phi_{\tau}^{*}\left(\left.\theta_{L}\right|_{u}\right)=\left.\theta_{L}\right|_{v}$, where $u=\beta(\tau)$ and $v=\alpha(\tau)$.

Let $J_{1}: X_{1} \rightarrow P$ and $J_{2}: X_{2} \rightarrow P$ be any two symplectic realizations of $P$, on which the symplectic groupoid $\left(\Gamma \rightrightarrows P, \alpha_{0}, \beta_{0}\right)$ acts freely and properly. Suppose that $\phi_{1}: L_{1} \rightarrow X_{1}$ and $\phi_{2}: L_{2} \rightarrow X_{2}$ are prequantum bundles with connection forms $\theta_{L_{1}}$ and $\theta_{L_{2}}$, respectively. It follows from Lemma 6.1 that $E \backslash\left(\overrightarrow{L_{2}} * L_{1}\right)$, where the groupoid $E$ acts on $\left(\overline{L_{2}} * L_{1}\right)$ diagonally, is a circle bundle over $\Gamma \backslash\left(\overline{X_{2}} * X_{1}\right)$.

Theorem 6.1. The one-form $\left(-\theta_{L_{2}}, \theta_{L_{1}}\right)$ descends to a connection form on the circle bundle

$$
\left.\left.E \backslash \overline{\left(L_{2}\right.} * L_{1}\right) \rightarrow \Gamma \backslash \overline{\left(X_{2}\right.} * X_{1}\right),
$$

which is in fact a prequantum bundle.

Proof. According to Lemma 6.2, both $\theta_{L_{1}}$ and $-\theta_{L_{2}}$ are invariant under the groupoid $E$-action. To show that $\left(-\theta_{L_{2}}, \theta_{L_{1}}\right)$ descends to the quotient space $E \backslash\left(\overline{L_{2}} * L_{1}\right)$, it suffices to show that the restriction of $\left(-\theta_{L_{2}}, \theta_{L_{1}}\right)$ to any diagonal $E$-orbit is zero.

Let $\widetilde{J}_{1}=J_{1} \circ \phi_{1}$ and $\tilde{J}_{2}=J_{2} \circ \phi_{2}$, and let $\zeta_{1}$ and $\zeta_{2}$ denote the Euler vector fields on $L_{1}$ and $L_{2}$, respectively. We note that the tangent space to the diagonal $E$-orbit 
at any $\left(z_{2}, z_{1}\right) \in \tilde{J}_{2}^{-1}(u) \times \tilde{J}_{1}^{-1}(u)$ is spanned by vectors of the form $\left(\hat{X}_{J_{2}^{*} \theta}\left(z_{2}\right)-f(u) \zeta_{2}, \hat{X}_{J_{1}^{*} \theta}\left(z_{1}\right)-f(u) \zeta_{1}\right)$, where $\theta \in \Omega^{1}(P)$ and $f \in C^{\infty}(P)$. It is simple to see that

$$
\begin{aligned}
& \left.\left(\hat{X}_{J_{2}^{*} \theta}\left(z_{2}\right)-f(u) \zeta_{2}, \hat{X}_{J_{1}^{*} \theta}\left(z_{1}\right)-f(u) \zeta_{1}\right)\right\lrcorner\left(-\theta_{L_{2}}, \theta_{L_{1}}\right) \\
& \left.\left.=-\left(\hat{X}_{J_{2}^{*} \theta}\left(z_{2}\right)-f(u) \zeta_{2}\right)\right\lrcorner \theta_{L_{2}}+\left(\hat{X}_{J_{1}^{*} \theta}\left(z_{1}\right)-f(u) \zeta_{1}\right)\right\lrcorner \theta_{L_{1}} \\
& =f(u)-f(u) \\
& =0 .
\end{aligned}
$$

Thus, $\left(-\theta_{L_{2}}, \theta_{L_{1}}\right)$ descends to a well defined one-form on the quotient space. It is simple to see that it is indeed a connection form defining a prequantum bundle.

As discussed in Sect. 4, if the Poisson manifold $P$ is a Poisson group $G^{*}$, any classical intertwiner space would reduce to the Poisson group reduction $J^{-1}\left(1_{G^{*}}\right) / G$ for a certain symplectic realization $J: X \rightarrow G^{*}$. In this case, the prequantum bundle can also be described in a simpler form.

Let $C$ be the level set $J^{-1}\left(1_{G^{*}}\right)$. Suppose that $\phi: L \rightarrow X$ is a prequantum bundle with connection $\theta_{L}$. Then, the map

$$
\left.\xi \in \mathfrak{g} \rightarrow \hat{X}_{\xi}\right|_{C} \in \mathscr{X}\left(\phi^{-1}(C)\right)
$$

is indeed a Lie algebra homomorphism since the intersection of $C$ with any $G$-orbit is isotropic. Here $\left.\hat{X}_{\xi}\right|_{C}$ denotes the horizontal lift on $\phi^{-1}(C)$ of the infinitesimal generator $X_{\xi}$ of the Lie algebra g-action. Therefore, Eq. (2) also defines a Lie group $G$-action on $\phi^{-1}(C)$ if $G$ is simply connected. However, we should note that, in general, it is impossible to lift the $G$-action to the entire bundle $L$.

Proposition 6.1. The pull back of the connection form $\theta_{L}$ on $\phi^{-1}(C)$ descends to a one-form on the quotient space $\phi^{-1}(C) / G$, which defines a prequantum connection on the circle bundle $\phi^{-1}(C) / G \rightarrow J^{-1}\left(1_{G^{*}}\right) / G$.

Proof. Let $E \stackrel{\pi}{\rightarrow} D$ be a prequantum bundle of the symplectic groupoid $\left(D \rightrightarrows G^{*}\right.$ $\left., \alpha_{0}, \beta_{0}\right)$, and $1 \in E$ any element over the point $\left\{1_{G}\right\} \times\left\{1_{G^{*}}\right\} \in D\left(\cong G \times G^{*}\right)$. Since the identity space $G^{*}$, considered as a lagrangian submanifold of $D$ (i.e., being identified with $\left\{1_{G}\right\} \times G^{*} \subseteq D$ ), is simply connected, it possesses a horizontal lift through the point $1 \in E$. Thus, according to Theorem $3.1, E$ possesses a groupoid structure which is a central extension of $D$. Since $\beta_{0}^{-1}\left(1_{G^{*}}\right) \cong G \times\left\{1_{G^{*}}\right\}$ is a simply connected lagrangian submanifold, the restriction of $E$ on $\beta_{0}^{-1}\left(1_{G^{*}}\right)$ can be naturally identified with the trivial bundle $G \times\left\{1_{G^{*}}\right\} \times T^{1}$.

The following lemma, which can be easily verified, describes explicitly how $E$ acts on $\phi^{-1}(C)$ under this trivialization.

Lemma 6.3. Under the isomorphism $\left.E\right|_{\beta_{0}^{-1}\left(1_{G^{*}}\right)} \cong G \times\left\{1_{G^{*}}\right\} \times T^{1}$ as described above, the groupoid E-action on $\phi^{-1}(C)$ is given by

$$
\left(g, 1_{G^{*}}, t\right) \cdot l=t(g l), \quad \forall g \in G, t \in T^{1}, l \in \phi^{-1}(C),
$$

where, on the right-hand side, gl denotes the group G-action on $\phi^{-1}(C)$ as defined by Eq. (2).

According to Theorem 6.1, the prequantum bundle over $J^{-1}\left(1_{G^{*}}\right) / G$ is $E \backslash\left(T^{1} \times \phi^{-1}(C)\right)$, which is isomorphic to $\phi^{-1}(C) / G$ according to Lemma 6.3. 
Under such an isomorphism, the connection form on $E \backslash\left(T^{1} \times \phi^{-1}(C)\right)$ goes to the natural one-form on $\phi^{-1}(C) / G$ induced from the one on $\phi^{-1}(C)$ obtained by pulling back $\theta_{L}$.

Remark. (1) When $G$ is a usual Lie group equipped with the zero Poisson structure, the dual group $G^{*}$ is the usual Lie-Poisson space $\mathfrak{g}^{*}$. Proposition 6.1 thus reduces to Theorem 3.2 in [5].

(2) The prequantum bundle $E$ has a double groupoid structure, corresponding to the double groupoid structure on $D$. In the meantime, $D$ has a group structure known as a double group. It would be quite interesting to know if this double groupoid structure on $E$ is related to some central extension of $D$ considered as a double group.

\section{Polarization}

A polarization on a symplectic manifold $S$ is an integrable lagrangian subbundle of $T^{\mathbb{C}} S$, the complexification of the tangent bundle $T S$. A polarization is real if it is contained in the real tangent bundle $T S$. In other words, a real polarization is a lagrangian foliation of the symplectic manifold $S$, i.e., a foliation in which each leaf is a lagrangian submanifold. Usually, polarization is the difficult part in carrying out a geometric quantization. This section is devoted to the problem of investigating what geometric data will naturally induce a polarization on the classical intertwiner space. For simplicity, we shall confine our attention to real polarizations. Nevertheless, we wish that our idea could be generalized to other types of polarizations as well.

We first start from the following:

Definition 7.1. Let $(\Gamma \rightrightarrows P, \alpha, \beta)$ be a symplectic groupoid and $J: X \rightarrow P$ a symplectic realization. Suppose that $\Gamma$ has a polarization $\mathscr{F}_{\Gamma}$. A polarization $\mathscr{F}_{X}$ on $X$ is said to be compatible with $\mathscr{F}_{\Gamma}$ iff the restriction of the foliation $\mathscr{F}_{\Gamma} \times \mathscr{F}_{X} \times \mathscr{F}_{X}$ to the graph of the action $\Lambda \subset \Gamma \times X \times X$ induces a foliation $\mathscr{F}_{\Lambda}$ on $\Lambda$, and the derivative of $p_{2}$ (which is the projection to the second factor) at any $z \in \Lambda$ maps $\left.\mathscr{F}_{\Lambda}\right|_{z}$ surjectively onto $\left.\mathscr{F}_{X}\right|_{p_{2}(z)}$.

The following proposition gives a useful criterion for determining a compatible polarization.

Proposition 7.1. Let $(\Gamma \rightrightarrows P, \alpha, \beta)$ be a symplectic groupoid with a polarization $\mathscr{F}_{\Gamma}$. Let $J: X \rightarrow P$ be a symplectic realization with a polarization $\mathscr{F}_{X}$ such that the restriction of $\mathscr{F}_{\Gamma} \times \mathscr{F}_{X} \times \mathscr{F}_{X}$ to the graph of action $\Lambda \subset \Gamma \times X \times X$ is a foliation on $\Lambda$. $\mathscr{F}_{X}$ is a compatible polarization if for any $(r, x) \in \Gamma \times X$ such that $\beta(r)=J(x)$, we have

1. $\left.\left.T J \mathscr{F}_{X}\right|_{x} \subseteq T \beta \mathscr{F}_{\Gamma}\right|_{r} ;$ and

2. $\operatorname{Tm}\left(T_{(r, x)}(\Gamma * X) \cap\left(\mathscr{F}_{\Gamma} \times \mathscr{F}_{X}\right)\right) \subseteq \mathscr{F}_{X}$, where $m: \Gamma * X \rightarrow X$ denotes the groupoid action.

Proof. Let $z=(r, x, y)$ be any point in $\Lambda$. For any $\left.v_{x} \in \mathscr{F}_{X}\right|_{x}$, there is $\left.v_{r} \in \mathscr{F}_{\Gamma}\right|_{r}$ such that $T J v_{x}=T \beta v_{r}$. Hence, $\left(v_{r}, v_{x}\right) \in T_{(r, x)}(\Gamma * X)$. By assumption, $v_{y}=T m\left(v_{r}, v_{x}\right) \in$ $\left.\mathscr{F}_{X}\right|_{y}$. Therefore, $\left.\left(v_{r}, v_{x}, v_{y}\right) \in \mathscr{F}_{\Gamma}\right|_{z}$. So $T p_{2}:\left.\left.\mathscr{F}_{\Gamma}\right|_{z} \rightarrow \mathscr{F}_{X}\right|_{x}$ is surjective.

The symplectic groupoid for a Lie-Poisson space $\mathfrak{g}^{*}$ is $\left(T^{*} G \rightrightarrows \mathfrak{g}^{*}, \alpha, \beta\right)$. There is a natural polarization on $T^{*} G$, namely, the one by cotangent fibers. It is easy to see that a polarization on a realization $J: X \rightarrow \mathfrak{g}^{*}$, or equivalently a Hamiltonian 
$G$-space, is compatible in the sense of definition above is equivalent to saying that the polarization is $G$-invariant. Therefore, it is natural to consider our definition as a generalization of $G$-invariant polarizations in the groupoid setting.

This viewpoint can also be justified by the following:

Proposition 7.2. A compatible polarization on $X$ naturally induces a foliation (which could be singular in general) on the quotient space $\Gamma \backslash X$.

Proof. Let $Q=\Gamma \backslash X$, and $\rho$ be the natural projection from $X$ to $Q$. For any $q \in Q$, let $x \in X$ be any point over $q$ and $\left.\mathscr{F}_{Q}\right|_{q}=T \rho\left(\left.\mathscr{F}_{X}\right|_{x}\right)$. Suppose that $y$ is another point in the same fiber. Then, there is an element $r \in \Gamma$ such that $(r, x, y) \in \Lambda$, where $\Lambda$ is the graph of the groupoid action. For any $\left.v_{x} \in \mathscr{\mathscr { F }}_{X}\right|_{x}$, by Definition 7.1, there is $\left.v_{r} \in \mathscr{F}_{\Gamma}\right|_{r}$ and $\left.v_{y} \in \mathscr{F}_{X}\right|_{y}$ such that $\left(v_{r}, v_{x}, v_{y}\right)$ is tangent to the submanifold $\Lambda$. On the other hand, it is simple to see that $\rho \circ p_{2}=\rho \circ p_{3}$ holds on $\Lambda$, where $p_{2}$ and $p_{3}$ are projections from $\Lambda$ to $X$ defined by $p_{2}(r, x, y)=x$ and $p_{3}(r, x, y)=y$ respectively. Therefore, $T \rho v_{x}=T \rho v_{y}$. This shows that $\left.\left.T \rho \mathscr{F}_{X}\right|_{x} \subseteq T \rho \mathscr{F}_{X}\right|_{y}$. Reversing the order of $x$ and $y$, it follows that $\left.T \rho \mathscr{F}_{X}\right|_{x}=\left.T \rho \mathscr{F}_{X}\right|_{y}$. In other words, $\left.\mathscr{F}_{Q}\right|_{q}$ does not depend on the choice of elements in the fiber $\rho^{-1}(q)$. Thus this gives rise to a well-defined subbundle $\mathscr{F}_{Q}$ of $T Q$ (the dimension of $\mathscr{F}_{Q}$ may not be constant). To prove that $\mathscr{F}_{Q}$ is integrable, let $E_{1}, E_{2}$ be any its smooth sections. We take $\Xi_{1}$ and $\Xi_{2}$ to be any sections of $\mathscr{F}_{X}$ lifting $E_{1}$ and $E_{2}$ respectively, i.e., $E_{1}=T \rho \Xi_{1}$ and $E_{2}=T \rho \Xi_{2}$. Then, $\left[E_{1}, E_{2}\right]=T \rho\left[\Xi_{1}, \Xi_{2}\right]$ is also a section of this subbundle. Therefore, $\mathscr{F}_{Q}$ is integrable.

Theorem 7.1. Suppose that $(\Gamma \rightrightarrows P, \alpha, \beta)$ is a symplectic groupoid and $\mathscr{F}_{\Gamma}$ a polarization of $\Gamma$. Let $J_{1}: X_{1} \rightarrow P$ and $J_{2}: X_{2} \rightarrow P$ be realizations with compatible polarizations $\mathscr{F}_{1}$ and $\mathscr{F}_{2}$, respectively. Assume that the restriction of $\mathscr{F}_{2} \times \mathscr{F}_{1}$ on $\left(\overline{X_{2}} * X_{1}\right)$ is a well-defined foliation. Then, $\Gamma \backslash\left(\overline{X_{2}} * X_{1}\right)$ has an induced polarization.

Proof. It can be proved by the same argument as in the proof of Proposition 7.2 that the foliation $\mathscr{F}_{2} \times \mathscr{F}_{1}$ on $\left(\overline{X_{2}} * X_{1}\right)$ passes to a foliation $\mathscr{F}_{Q}$ on the quotient space $Q=\Gamma \backslash\left(\overline{X_{2}} * X_{1}\right)$. Let $\rho$ be the projection from $\overline{X_{2}} * X_{1}$ to $Q$. To show that it is a polarization, we note that for any $q=\rho\left(x_{2}, x_{1}\right)$, as a symplectic vector space the tangent space $T_{q} Q$ can be naturally identified with the quotient space $R / R^{\perp}$, where $R$ is the coisotropic subspace $T_{\left(x_{2}, x_{1}\right)}\left(\overline{X_{2}} * X_{1}\right)$ and $R^{\perp}$ its symplectic complement. Under such an identification, $\left.\mathscr{F}_{Q}\right|_{q}$ is then equal to $(L \cap R) /\left(L \cap R^{\perp}\right)$, where $L$ is the lagrangian subspace $\left.\mathscr{F}_{2}\right|_{x_{2}} \times\left.\mathscr{F}_{1}\right|_{x_{1}}$. It is known that $(L \cap R) /\left(L \cap R^{\perp}\right)$ is a lagrangian subspace of $R / R^{\perp}$, according to Sect. 3, Ch. 4 of [6]. This shows that $\mathscr{F}_{Q}$ is indeed a polarization.

\section{Discussion}

As we know, what makes Guillemin-Sternberg theory so interesting is essentially the fact that the geometric quantization procedure commutes with the reduction procedure. This fact enables us to relate various notions in representation theory with those in symplectic geometry [5]. A natural extension of this fact in our context should read as:

$$
\left.\operatorname{Quan}\left(\Gamma \backslash \overline{X_{2}} \times X_{1}\right)\right) \cong \operatorname{Hom}_{\mathscr{A}}\left(V_{2}, V_{1}\right),
$$


where $\mathscr{A}$ is an associative algebra quantizing the symplectic groupoid $\Gamma$, while $\rho_{1}: \mathscr{A} \rightarrow \operatorname{End}\left(V_{1}\right)$ and $\rho_{2}: \mathscr{A} \rightarrow \operatorname{End}\left(V_{2}\right)$ are the representations obtained by quantizing the symplectic realizations $J_{1}: X_{1} \rightarrow P$ and $J_{2}: X_{2} \rightarrow P$ respectively. However, in practice, due to the lack of a general machinery, it is difficult to quantize a symplectic groupoid to an associative algebra except for some examples $[22,23,20]$. We note that when the symplectic groupoid $\Gamma$ is $\left(T^{*} G \rightrightarrows \mathfrak{g}^{*}, \alpha, \beta\right)$, the universal enveloping algebra $U \mathfrak{g}$, or the convolution algebra of $G$, can be considered as the corresponding quantized associative algebra $\mathscr{A}$, so the right-hand side of Eq. (3) essentially becomes $\operatorname{Hom}_{G}\left(V_{2}, V_{1}\right)$ and therefore Guillemin-Sternberg theory is indeed a special case of Eq. (3).

Although it seems unlikely that there exists a general method producing associative algebras out of symplectic groupoids, there is a large class of interesting algebras which is believed to be produced in such a way. Those are, namely, quantum groups as mentioned in the introduction. A basic example is the symplectic groupoid over the dual Poisson Lie group $G^{*}$ of a Bruhat Poisson Lie group [13] $G$. There are however at least two major difficulties in order to carry this out. While the symplectic groupoid $\left(T^{*} G \rightrightarrows \mathrm{g}^{*}, \alpha, \beta\right)$ has a canonical polarization, namely, the foliation by cotangent fibers, which is in fact the key ingredient that makes Guillemin-Sternberg theory much simpler, it is not clear if there exists a natural polarization for the symplectic groupoid $\left(D \rightrightarrows G^{*}, \alpha, \beta\right)$ of $G^{*}$ (see [12] for more detail on this groupoid). On the other hand, quantizing a realization needs a polarization on $X$ and for compact $X$ the polarization is usually taken to be positive-definite, i.e., a Kähler structure on $X$. For a Bruhat Poisson group $G$, basic elements of realizations are those symplectic leaves of $G^{*}$, which are the so-called dressing orbits. However, it is not known if a dressing orbit of $G^{*}$ admits a natural Kähler structure. Of course, abstractly such a Kähler structure always exists as a consequence of recent work of Ginzburg and Weinstein [4]. The problem is whether there is a natural way of describing this Kähler structure, and perhaps more importantly, whether such a Kähler polarization is compatible with the polarization on the groupoid $D$ itself. Whether we can extend Guillemin-Sternberg theory successfully to this general context highly depends on to what extent these difficulties could be solved.

\section{References}

1. Bott, R., Tu, L.W.: Differential forms in algebraic topology. GTM 82, Berlin Heidelberg New York: Springer 1982

2. Coste, A., Dazord, P., Weinstein, A.: Groupoïdes symplectiques. In: Publications du Département de Mathématiques de l'Université de Lyon, I, number 2/A-1987, 1987, pp. 1-65

3. Drinfel'd, V.G.: Quantum groups. In: A.M. Gleason (ed.) Proceedings of the International Congress of Mathematicians, Berkeley, 1986, Providence, RI: American Mathematical Society, 1987 , pp. $798-820$

4. Ginzburg, V., Weinstein, A.: Lie-Poisson structure on some Poisson Lie groups. J. of AMS 5, No. 2, 445-453 (1992)

5. Guillemin, V., Sternberg, S.: Geometric quantization and multiplicities of group representations. Invent. Math. 67, 515-538 (1982)

6. Guillemin, V., Sternberg, S.: Geometric asymptotics. Volume 14, Mathematical Surveys and Monographs. American Mathematical Society, revised edition, 1990

7. Higgins, P.J., Mackenzie, K.C.H.: Algebraic constructions in the category of Lie algebroids. J. Algebra 129, 194-230 (1990) 
8. Huebschmann, J.: Poisson cohomology and quantization. J. Reine Angew. Math. 408, 57-113 (1990)

9. Kirillov, A.A.: Elements of the theory of representations. Volume $\mathbf{2 2 0}$ of Grundlehren der mathematischen Wissenschaften. Berlin Heidelberg New York: Springer, 1976

10. Landsman, N.P.: Rieffel induction as generalized quantum Marsden-Weinstein reduction. Preprint, 1993

11. Jiang-Hua Lu.: Momentum mappings and reduction of Poisson actions. In: P. Dazord, A. Weinstein (eds.) Symplectic geometry, groupoids, and integrable systems, Seminaire sud Rhodanien a Berkeley, 1989, Berlin Heidelberg New York: Springer-MSRI publications, 1991, pp. 209-226

12. Jiang-Hua Lu, Weinstein, A.: Groupoïdes symplectiques doubles des groupes de Lie-Poisson. C.R. Acad. Sci. Paris Sér. I Math. 309, 951-954 (1989)

13. Jiang-Hua Lu, Weinstein, A.: Poisson Lie groups, dressing transformations, and Bruhat decompositions. J. Differential Geom. 31, 501-526 (1990)

14. Mackenzie, K.C.H.: Lie groupoids and Lie algebroids in differential geometry. London Mathematical Society Lecture Note Series, no. 124. Cambridge: Cambridge University Press, 1987

15. Mackenzie, K.C.H., Xu, P.: Lie bialgebroids and Poisson groupoids. Duke Math. J., 73 No. 2, 415-452 (1994)

16. Marsden, J., Weinstein, A.: Reduction of symplectic manifolds with symmetry. Rep. Math. Phys. 5, 121-129 (1974)

17. Mikami, K., Weinstein, A.: Moments and reduction for symplectic groupoids. Publ. Res. Inst. Math. Sci. 24, 121-140 (1988)

18. Semenov-Tian-Shansky, M.A.: Dressing transformations and Poisson group actions. Publ. Res. Inst. Math. Sci. 21, 1237-1260 (1985)

19. Sniatycki, J.: Geometric quantization and quantum mechanics. Berlin Heidelberg New York: Springer, 1980

20. Szymczak, I., Zakrzewski, S.: Quantum deformations of Heisenberg group obtained by geometric quantization. J. Geom. Phys. 7, N. 4, 553-569, (1990)

21. Weinstein, A.: Symplectic groupoids and Poisson manifolds. Bull. Am. Math. Soc. (N.S.) 16, 101-104 (1987)

22. Weinstein, A.: Noncommutative geometry and geometric quantization. In: J. Elhadad, P. Donato, D. Duval and G.M. Tuynman, editors, Symplectic geometry and mathematical Physics, Seminaire sud-rhodanien de géométrie, Aix-en-Provence 1990, Basel Boston: Birkhäuser, Progress in Mathematics, number 99, 1991, pp. 447-461

23. Weinstein, A.: Symplectic groupoids, geometric quantization, and irrational rotation algebras. In: P. Dazord and A. Weinstein, editors, Symplectic geometry, groupoids, and integrable systems, Seminaire sud Rhodanien a Berkeley, 1989, Berlin Heidelberg New York: Springer-MSRI publications, 1991, pp. 281-290

24. Weinstein, A., Xu, P.: Extensions of symplectic groupoids and quantization. J. Reine Angew. Math. 417, 159-189 (1991)

25. Weinstein, A., Xu, P.: Classical solutions to the quantum Yang-Baxter equation. Commun. Math. Phys. 148, 309-343 (1992)

26. Xu, P.: Morita equivalence of Poisson manifolds. Commun. Math. Phys. 142, 493-509 (1991)

27. Xu, P.: Morita equivalent symplectic groupoids. In: P. Dazord, A. Weinstein (eds.) Symplectic geometry, groupoids, and integrable systems, Seminaire sud Rhodanien a Berkeley, 1989, Berlin Heidelberg New York: Springer-MSRI publications, 1991, pp. 291-311

28. Xu, P.: Morita equivalence and symplectic realizations of Poisson manifolds. Ann. Scient. Éc. Norm. Sup., 4 série, 25, 307-333 (1992)

29. Xu, P.: Symplectic groupoids of reduced Poisson spaces. C. R. Acad. Sci. Paris. Sér. I. Math., 314, 457-461 (1992)

Communicated by N.Yu. Reshetikhin 